\title{
Time Series Forecasting Model Based on Wavelet Denoising Application in Manufacturing PMI Prediction
}

\author{
Dongdong $\mathrm{Bai}^{1,}$, , Jinyu Wei ${ }^{2, \mathrm{~b}}$ \\ ${ }^{1}$ Tianjin University of Technology, Tianjin 300384, China \\ 2 Tianjin University of Technology, Tianjin 300384, China \\ a1084745728@qq.com, bweijinyu2010@126.com
} Keywords: wavelet denoising; ARIMA model; Manufacturing PMI; Prediction interval; Time series
analysis.

\begin{abstract}
PMI is of great significance in terms of business analysis and economic forecasts. To accurately grasp the running of macroeconomic trend the right strategic decisions and auxiliary enterprises, this article takes our country manufacturing PMI as the research object, the wavelet noise reduction as the core, combined with the ARIMA model is established based on the wavelet noise reduction of ARIMA non-stationary time series forecasting model. Finally it used the model for the empirical analysis of China's manufacturing PMI. The results show that the prediction precision of the model, fitting and prediction intervals are improved obviously. Our country economy is in steady stage, our country macroeconomic policy has obvious effect
\end{abstract}

\section{Introduction}

Wavelet Analysis is proposed by French engineer J.M orlet the first time in $1974^{[1]}$.After years of development, the wavelet analysis has become a common method of time-frequency analysis ${ }^{[2]}$.Has the characteristics of multi-resolution analysis of wavelet analysis, can characterize local signal in time domain and frequency domain information, time and frequency window can be adjusted according to the concrete forms of signal dynamic, in general, in the low frequency part of signal (more stable) can use lower temporal resolution, and improve the resolution of frequency, under the condition of high frequency (frequency little change) at a low frequency resolution can be used for accurate positioning ${ }^{[3]}$.Therefore, wavelet analysis can detect the transient components in the normal signal, is called "mathematics microscope", is widely used in various time-frequency analisis ${ }^{[4]}$.

PMI (Purchasing Managers Index) is a system of comprehensive economic indicator released monthly, PMI covers production and circulation, in areas such as manufacturing and non-manufacturing, divided into manufacturing PMI, services PMI, etc. Manufacturing PMI originating in the United States in the 1930 s, after decades of development, the manufacturing PMI (purchasing managers' index) has become a measure of manufacturing industry in production, new orders, commodity price, inventory, employee, order delivery and new export orders and the index of eight aspects such as the import conditions ${ }^{[5]}$.PMI index for 50 from watershed. When the PMI is greater than 50, the economy in the development of the PMI index more than 50 shows that economic development more strong; When the PMI is less than 50, the economy in recession, less than 50 shows that the recession faster and faster ${ }^{[6]}$.Through the numerical and trends of PMI, can grasp the current economic operation in the economic cycle of the stage, timely find possible turning point in the economy, insight into economic internal structure change, and through the comparative analysis to predict the future economic might. Enterprise can also through the PMI understanding the operating state of the economy as a whole and direction ${ }^{[7]}$.

Based on the principle of wavelet de-noising, the time series after noise reduction $\operatorname{ARIMA}(5,1,0)$ model is established, the projections for China's manufacturing PMI. It is of great significance to grasp the economic situation in our country and enterprises to make accurate real-time decision-making. 


\section{ARIMA Time Series Model Based on Wavelet De-noising Theory}

In the historical observation data collection, group 1 to $j$ of data as the observation data, the group $j+1$ to $n$ of data as test data, used to detect the precision of the prediction model and fitting.As the original time series signal and superscript 0 indicates the decomposition scale, at this point, the 0 scale time series as the original time series. And then select the scale function $\phi(t)$ and wavelet basis function of $N$ scale decomposition, set high frequency detail coefficients and a set of low-frequency approximate coefficient. In order to take into account the smoothness of wavelet de-noising and two similarity criterion, the selection of threshold $\delta$ on the global threshold to determine the threshold, is a combination of Stein unbiased likelihood estimation based (SURE) soft threshold estimation and the length of the logarithm of threshold of heuristic SURE threshold. Threshold effect in the sequence of each coefficient, the modulus value is less than the coefficient is set to 0 , only keep modulus value is greater than $\delta$, namely:

$$
c_{\delta}=\left\{\begin{array}{l}
c,|c|>\delta \\
0
\end{array}\right.
$$

Sequence signals and then get the set time, to get new sequence and wavelet reconstruction, and get the time series after noise reduction. Using ARIMA time series model regression prediction to get sequence.

\section{The Time Series Model Based on Wavelet De-noising PMI Predictions}

\subsection{Wavelet Decomposition of the Original Sequence}

This paper adopts between January 2013 and September 2013 in China's manufacturing PMI as raw data, which in January 2013 to August 2014 PMI value for observation data, the value of the PMI in September 2014 for the testing data. To standardize the original observation data, the first with Daubechies wavelet as the wavelet basis function to standardized data after wavelet decomposition. Select db3 wavelet basis function, to 3 scale decomposition of standardized data, three groups of high frequency detail coefficients and a set of low frequency approximation coefficient.

\subsection{The Wavelet Denoising}

After wavelet decomposition of three groups of high frequency detail coefficients for global threshold quantization process, get a 3 set of new high frequency detail sequence, and details of high frequency and low frequency approximation sequences of wavelet reconstruction after get noise signal sequence, thus inhibiting the useless part of the signal, to restore effective information in the signal, as shown in Fig.1.
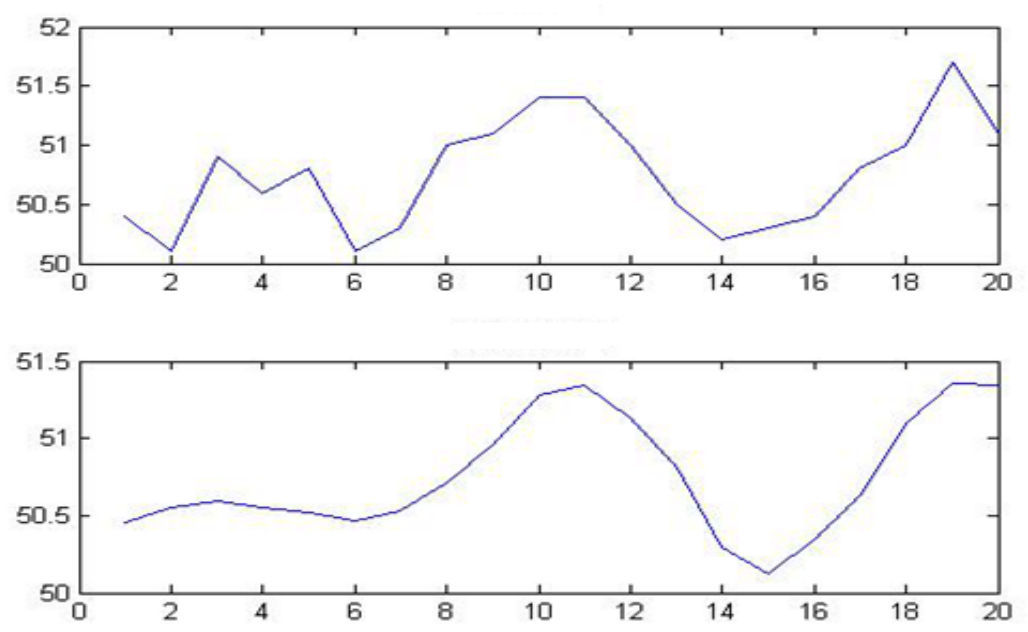

Fig. 1 original signal and denoising signal 


\subsection{ARIMA Model of Denoising Signal}

The structure of the ARIMA model identification is mainly using ACF autocorrelation coefficient and partial correlation coefficient PACF sequence estimation model of general categories, etc. Order autoregressive process for ACF is gradually decay exponential relationship, called the tail, and its PACF truncated after the interval; accordingly, order moving average process for ACF truncated after the interval, and PACF trailing.

ARIMA model to the residuals from the correlation test and information criterion method is adopted to identify, through SPSS comparison recognition, think that the best model of ARIMA $(5,0)$, its $R^{2}=0.958$, smooth $R^{2}=0.891$. As shown in Table 1 for ARIMA $(5,1,0)$ model of the actual value compared with the predicted value.

Table 1 the actual value compared with the predicted value

\begin{tabular}{|c|c|c|c|}
\hline month & actual value & predicted value & redisual \\
\hline 2013.1 & 50.4 & 50.4 & 0 \\
\hline 2013.2 & 50.1 & 50.4 & -0.3 \\
\hline 2013.3 & 50.9 & 50.6 & 0.3 \\
\hline 2013.4 & 50.6 & 50.6 & 0 \\
\hline 2013.5 & 50.8 & 50.5 & 0.3 \\
\hline 2013.6 & 50.1 & 50.5 & -0.2 \\
\hline 2013.7 & 50.3 & 50.4 & -0.1 \\
\hline 2013.8 & 51.0 & 50.7 & 0.3 \\
\hline 2013.9 & 51.1 & 50.9 & 0.2 \\
\hline 2013.10 & 51.4 & 51.2 & 0.2 \\
\hline 2013.11 & 51.4 & 51.5 & -0.1 \\
\hline 2013.12 & 51.0 & 51.3 & -0.3 \\
\hline 2014.1 & 50.5 & 50.9 & -0.4 \\
\hline 2014.2 & 50.2 & 50.4 & -0.2 \\
\hline 2014.3 & 50.3 & 50.1 & 0.2 \\
\hline 2014.4 & 50.4 & 50.3 & 0.1 \\
\hline 2014.5 & 50.8 & 50.7 & 0.1 \\
\hline 2014.6 & 51.0 & 51.1 & -0.1 \\
\hline 2014.7 & 51.7 & 51.4 & 0.3 \\
\hline 2014.8 & 51.1 & 51.3 & -0.2 \\
\hline
\end{tabular}

\subsection{Accuracy Evaluation}

For the model can predict the change characteristics of PMI, must carry on the model test.This article selects the value of detection of the PMI in September 2014 variables, of ARIMA time series prediction model and ARIMA time series forecasting model based on wavelet noise reduction.The results are shown in Table 2.

Table 2 prediction accuracy

\begin{tabular}{|c|c|c|c|}
\hline Test index & ARIMA modle & $\begin{array}{c}\text { ARIMA model based on } \\
\text { wavelet denoising }\end{array}$ & \multirow{2}{*}{ actual value } \\
\cline { 1 - 3 }$R^{2}$ & 0.373 & 0.958 & \\
\hline UCL & 52.1 & 51.4 & \\
\hline LCL & 50.3 & 51.0 & \multirow{2}{*}{51.1} \\
\hline predicted value & 51.5 & 51.2 & \\
\hline redisual & -0.4 & -0.1 & \\
\hline
\end{tabular}

\section{Summary}

The ARIMA model based on wavelet noise reduction in China's PMI has achieved good effect. Combining wavelet noise reduction and ARIMA model to build the combination forecast model predicted results and directly using the predictive results of the ARIMA model is used in the comparison. The results showed that the ARIMA model based on wavelet noise reduction degree of 
fitting is better, higher prediction precision, smaller residual, and ARIMA model prediction based on wavelet noise reduction prediction intervals smaller, to China's macroeconomic forecasting and monitoring is of important practical reference value; PMI in a slightly larger than 50 trend in our country, suggests that our country is in the steady development of economy.

\section{References}

[1] Dong Changhong. Matlab wavelet analysis toolbox principle and application [M] Beijing: national defence industry press, 2004:108-125.

[2] Zhang Libin Feng Yi. China PMI empirically the relationship between GDP [J] statistics and decision making. 2012 (2): 143-145.

[3] Wang Jiayi Yao Jian. Combined with PMI forecast analysis of investment in fixed assets financial economy [J]. 2012:124-126.

[4] zhang ning, Chen Qing Yi, zhou li. Based on the purchasing managers' index of China's economic growth forecast indicators analysis [J] currency theory. 2013, 5:19-22.

[5] zhi-yuan wang, the mo. PMI and GDP and an empirical analysis of the Shanghai industrial index correlation [J]. Journal of chongqing institute of technology, 2014 (7): 69-71.

[6] zhao-rong Chen. China's manufacturing PMI index prediction index, based on the analysis of the ARIMA model [J]. 2014 (5): 78-79.

[7] li na. China's manufacturing purchasing managers' index (PMI) introduction [J] China statistics. 2013 (12): 23 and 24. 